\title{
Orientierende Untersuchungen zur elektrokinetischen Sicherung kontaminierter Standorte
}

\author{
Prof. Dr.-Ing. Udo Hellwig, Dr. Harry Jehring, Dipl.-Geophys. Edgar Wetzig
}

\section{Einleitung}

Forschungsprojekte aus den USA und den Niederlanden haben die vielseitige Einsetzbarkeit der Elektrokinetik bereits bewiesen. Erfolgreich eingesetzt wurde die Methode $\mathrm{z}$. B. in den USA als Präventivmaßnahme zum Schutz des Grundwassers im An- und Abstrombereich von Deponien. Weitere typische Anwendungsgebiete für die Elektrokinetik sind:

- schwermetallkontaminierte Standorte allgemein

- schwierig zu dekontaminierende Standorte (z. B. wegen Überbauung)

- galvanotechnische Betriebe (alt und bestehend)

- Feuerverzinkereien

- bleiverarbeitende Industrie

- Klärschlämme (Abwässer)

- Rieselfelder (Nitratbelastung)

- Galvanikschlämme (Schwermetalle)

- Hafenschlämme

- Limnische Schlämme

\subsection{Geschichtliches}

Auf dem Gelände der Technischen Fachhochschule (TFH) Wildau befanden sich (nacheinander) eine Essigfabrik, Betriebe der Holzverkohlungsindustrie (HIAG) sowie eine Gold- und Silberscheideanstalt. Von ca. 1900-1950 wurden hier chemische Produkte (Methano, Aceton, Blausäure, unbekannte Kampfstoffe etc.) hergestellt und abgelagert. Während des 2 . Weltkriegs wurden Sprengstoff und kriegswichtige Chemikalien hergestellt.

\subsection{Gegenwärtige Situation}

Beprobungen von Boden und Grundwasser auf dem Gelände der TFH Wildau ergaben Schadstoffkonzentrationen, die die Grenz-/Richtwerte zum Teil um mehr als das 1000-fache übersteigen. Die größten bisher festgestellten Arsenkonzentrationen betragen $51.000 \mu \mathrm{g} / \mathrm{l}$ (Grenzwert $60 \mu \mathrm{g} / \mathrm{l}$ ); an dieser Stelle ist die elektische Leit6fähigkeit des Grundwassers größer als $20.000 \mu \mathrm{S} /$ $\mathrm{cm}$ (Normalwerte liegen zwischen 500 und $1.33 \mu \mathrm{S} / \mathrm{cm}$ ). Da insbesondere Arsen in Boden und Grundwasser sehr mobil ist, ist davon auszugehen, daß das Grundwasser in einem größeren Bereich kontaminiert ist. Die Quellorte des Schadstoffeintrags sind z. Z. noch nicht hinreichend erfaßt. Dazu wären z. B. flächendeckende geophysikalische Kartierungen erforderlich.

Vorbereitend dazu wurde im Rahmen des beantragten Projekts eine geophysikalische Untersuchung des Flurstücks 480/2 (ehemaliges Sportplatzgelände) auf dem
Gelände der TFH Wildau durchgefuihrt. Aus den Untersuchungen geht ein unmittelbarer Handlungsbedarf hervor.

\section{Physikalisch-chemische Grundlagen}

Ionen und andere elektrisch geladene Teilchen erfahren in einem elektrischen Feld eine Kraft (Ionen-Migration). Sie werden beschleunigt und bewegen sich je nach Ladung in Richtung Anode oder Kathode. Abhängig von den Reibungsverlusten im Boden oder Wasser stellt sich ein Kräftegleichgewicht und damit eine bestimmte Driftgeschwindigkeit ein. Der Schadstofftransport erfolgt zusätzlich noch durch die sogenannte Elektro-Osmose: Wassertransport über die Einwirkung des elektrischen Feldes auf die elektrisch geladene Phasengrenze zwischen Mineral und Wasser (Elektrochemische Doppelschicht).

Arsen- und Schwermetallionen wandern z. T. in bestimmten Verbindungen mit der Kationen- bzw. Anionenfront in Richtung Anode bzw. Kathode, fällen teilweise als Hydroxidverbindungen auf ihrem Wege im Sanierungsraum aus und/oder scheiden sich (metallisch) an der Kathode ab; oder sie werden in einem kathodischen Brunnen angereichert und abgepumpt. Die Entsorgung der Elektroden bzw. schadstoffhaltigen Flüssigkeiten muß gesondert diskutiert werden. Die Ionen-Konzentrationen im Bereich der Elektroden werden so groß, daß die elektrokinetische Bodensanierung ggf. sogar auch als Rohstoffgewinnung im Sinne eines Recycling-Verfahrens angesehen werden kann.

Das Heraus- bzw. Ablösen der Schwermetalle von der Kornmatrix kann durch die Zugabe von ökologisch vertretbaren Säuren (z. B. organische Essigsäure $\mathrm{CH}_{3} \mathrm{COOH}$ ) in umweltverträglichen Konzentrationen ohne zusätzlichen Energieaufwand deutlich gesteigert werden.

Im Gegensatz zur Elektrokinetik funtionieren z. B. hydraulische Hochdruck-Bodenwaschanlagen nur bis zu einem gewissen Grad an Schluffanteilen im Boden. Bei diesen Verfahren darf der Anteil der kleinen Korngrößenfraktion einen bestimmten Grad nicht überschreiten. Anders bei der Elektrokinetik: Boden mit kleinem $\mathrm{K}_{\mathrm{f}}$ Wert haben größere relative Oberflächen, an denen in der Regel auch mehr Ladungsträger haften.

\subsection{Laufende Versuche an der TFH Wildau}

Seit Anfang Juni 1994 werden durch den Fachbereich Verfahrens- und Umwelttechnik der TFH Wildau Laborversuche zur elektrokinetischen Sanierung arsen- und schwermetallbelasteter Böden durchgeführt. Ziel dieser 
Versuchsreihe ist es, Aussagen darüber zu erhalten, welche umweltverträglichen Konditionierungsmaßnahmen für den Boden die Effizienz der elektrokinetischen Sanierung besonders steigern. Darüber hinaus ist der Einfluß von Aufbau und geometrischer Anordnung der Elektroden zu klären.

Die Untersuchungen werden z. Z. an einer gut geeigneten Bodenprobe von einer Erdstoffhalde bei Magdeburg mit extrem hohen Gehalten an Arsen, Cadmium, Blei und Zink durchgefuihrt. Es handelt sich um eine Serie wissenschaftlich-empirischer Versuche, mit deren Hilfe u. a. der Zusammenhang zwischen geophysikalischen Parametern wie der scheinbare spezifische Widerstand $r_{s}$ bzw. die spezifische elektrische Bodenleitfähigkeit s, ihrer relativen Änderungen und dem tatsächlichen Austrag an Metallionen (Schadstoffen) geklärt werden soll. In diesem Zusammenhang wird eine große Zahl (relativ aufwendiger) chemischer Bodenanalysen durchgefuihrt. Erste Erfolge und wichtige Erkenntnisse auch an der nicht wassergesättigten Probe sind bereits zu verzeichnen.

\section{Bodenproben}

Es war geplant, eine Bodenprobe vom Gelände der Technischen Fachhochschule Wildau im Labor zu untersuchen. Grundwasseranalysen hatten sehr hohe Arsengehalte ergeben; damit einhergehend wurden extrem hohe elektrische Leitfähigkeiten des kontaminierten Grundwassers festgestellt. Es bot sich daher an, den Quellort der Arsenbelastung und damit eine geeignete Position für eine Bodenprobe zunächst z. B. mittels einer geoelektrischen Kartierung zu bestimmen. Bevor damit begonnen wurde, erhielt die TFH Wildau

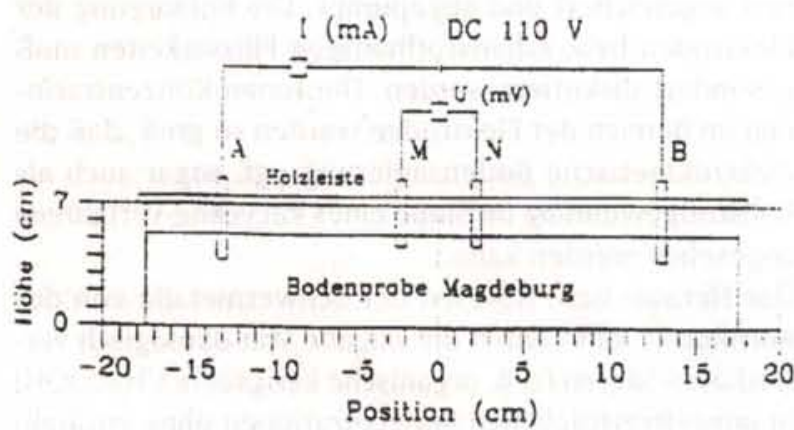

Abb. 1: Schematische Profilansicht bzgl. der Breite des Experimentiergefäßes; Meßsonden und Elektroden in Schlumbergeranordnung zur Bestimmung des spezifischen Widerstands $\rho$ in Wm eine Anfrage von der Sanierungsgesellschaft Magdeburg (SGM) hinsichtlich des zu erwartenden Erfolges bei der elektronkinetischen Sanierung schwermetallbelasteter Böden. Die SGM stand vor dem Problem, eine Entscheidung darüber treffen zu müssen, auf welche Weise eine mit Schwermetallen stark belastete Erdstoffhalde am aufwand- und kostengünstigsten zu sanieren sei.

Als Hilfe zur Beantwortung dieser Fragestellung bot die TFH an, eine Pilot-Laborstudie zunächst an einer Probe der Erdstoffhalde durchzufuihren. Die Experimente mit der Bodenprobe „Magdeburg“ sind als Vorversuche zu bewerten, mit deren Hilfe im Vorfeld der Arbeiten mit dem arsenbelasteten Boden in Wildau bestimmte Fragen z. B. zum Material- und Aufbau der Elektroden geklärt werden sollten.

\begin{tabular}{|c|c|c|c|c|}
\hline Teufe $(\mathrm{m})$ & Arsen (As) & Blei (Pb) & Cadmium (Cd) & Zink (Zn) \\
\hline $0-1$ & 5,49 & 399 & 5,5 & 503 \\
\hline $1-2$ & 126 & 3320 & 110 & 9470 \\
\hline $2-3$ & 85.5 & 2490 & 66,3 & 5810 \\
\hline $3-4$ & 158 & 6500 & 69,8 & 8670 \\
\hline $4-4,80$ & 287 & 12100 & 236 & 20100 \\
\hline \multicolumn{5}{|c|}{ Richtwerte holländische Liste (") } \\
\hline A-Wert & 20 & 50 & 1 & 200 \\
\hline B-Wert & 30 & 150 & 5 & 500 \\
\hline C-Wert & 50 & 600 & 20 & 3000 \\
\hline
\end{tabular}

Tabelle 1: Schwermetallbelastung in mg pro kg Trockensubstanz Probe der Rammkernsondierung RKS 8 unter Berücksichtigung der Tiefenstufe

$\left({ }^{\circ}\right)$ Die sog. Holländische Liste wurde erstmals 1983 für kontaminierte Standorte in den Niederlanden als Orientierungshilfe bei der Einschätzung von gemessenem Schadstoff, gehalten in Boden und Grundwasser, festgelegt:

$\mathrm{A}:=$ Referenzkategorie

$\mathrm{B}:=$ Kategorie für nähere Untersuchungen

C: $=$ Kategorie für Sanierungsuntersuchungen

\section{Versuchsdurchführung}

Bei allen Versuchen wurde mit einer konstanten Spannung von $18 \mathrm{~V}$ entsprechend $36 \mathrm{~V} / \mathrm{m}$ gearbeitet. Die Versuche wurden unterhalb der Zersetzungsspannung durchgefuihrt, um die Bildung des stark toxischen Arsenoxids zu verhindern. Diese Gasbildung würde dazu führen, daß sich ein Teil des Arsenaustrags der Massenbilanz entziehen würde. Zudem kommt es zu einer unzulässi-

IJC 40 (Volt)

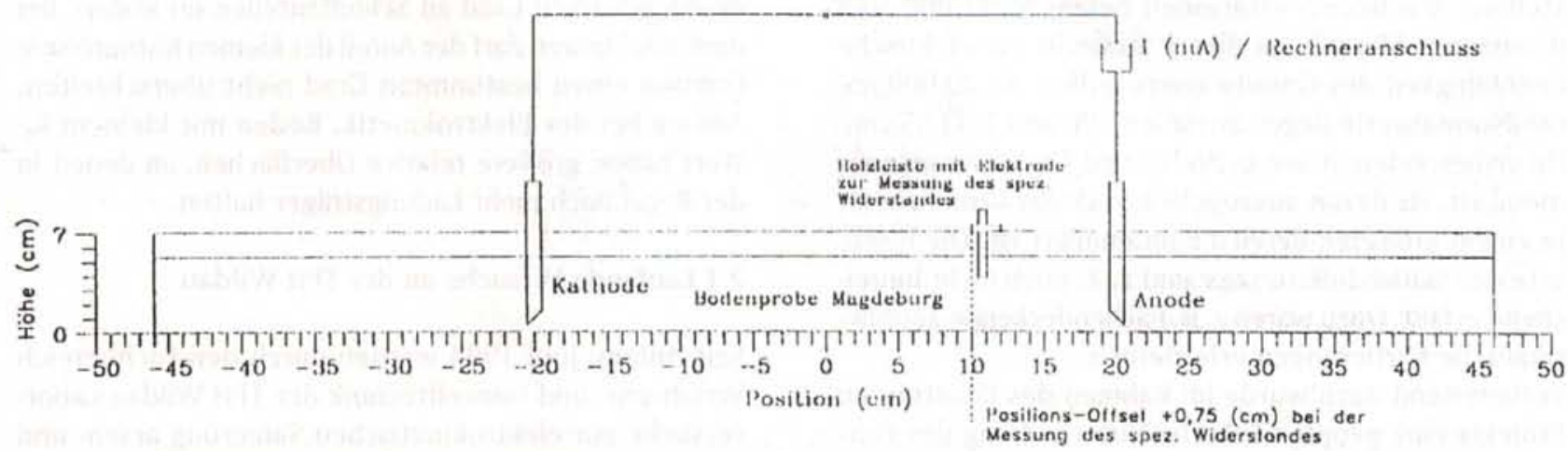

Abb. 2: Schematische Profilansicht bzgl, der Längsseite des Behälters; Positionen der Elektrokinetik-Elektroden am Beispiel der Volleisenelektroden $\emptyset 10$ mm 


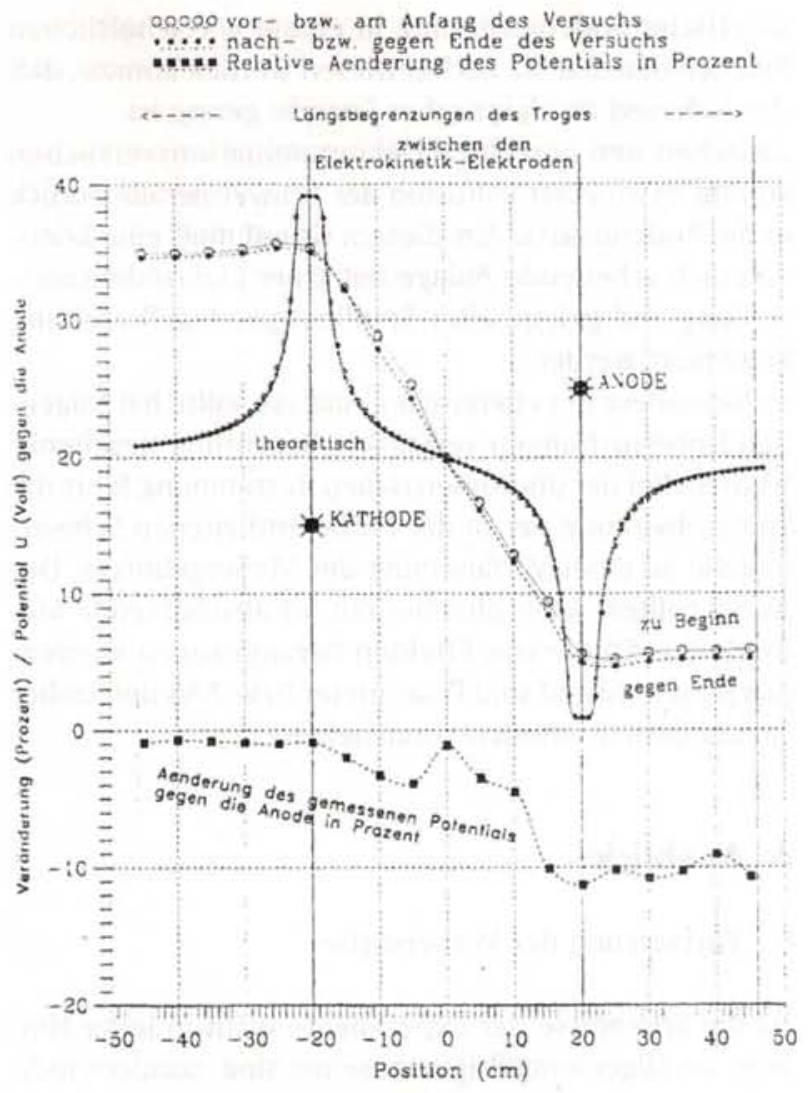

Abb. 3: Verlauf der Potentialdifferenz $\Delta U$ in der Bodenprobe gemessen gegen die Anode zu Beginn und gegen Ende von Elektrokinetik-Experiment 3; Änderung $\Delta U$ in \% Bodenprobe Magdeburg, feucht, massive, zylindrische EdelstahlElektroden $\emptyset 10 \mathrm{~mm}$ TFH Wildau, 04.07

gen Arbeitsplatzbelastung des Bedienungspersonals. Neben der Spannung wurde bei allen Versuchen der sich einstellende Strom aufgenommen, um die eingetragene Energiemenge zu bestimmen.

Es wurde bei allen Versuchen an einer wassergesättigten Probe gearbeitet, auf der sich partiell eine dünne Flüssigkeitsschicht ausbildete.

Um die As- bzw. Cd-Konzentrationsverteilung im Behälter zu bestimmen, wurden zu Versuchsbeginn (A) bzw. Versuchsende (E) Flüssigkeitsproben aus den Elektroden- taschen und dem Brunnen gezogen. Die Bestimmung der jeweiligen Konzentrationen erfolgte nach dem Schnelltestverfahren der Firma Merck (Merckoquant 10026/ Merck Cd 14834) - siehe Abschnitt 4. Verluste an Bodenwasser durch Probenahmen und Verdunstung wurden durch ganzflächige Aufgabe von Wasser bzw. Essigsäure kompensiert.

Bei den Vorversuchen stellte sich heraus, daß bei kurzen Versuchszeiten (ca. 2 h) keine signifikante Dekontamination der Bodenproben erzielt werden kann. Aus diesem Grund wurden die Versuche über einen Zeitraum von ca. 6 h durchgeführt. Aus sicherheitstechnischen Gründen war leider kein kontinuierlicher Betrieb (24 h) der Anlage möglich.

\section{Zusammenfassung}

Physikalische Ergebnisse

Deutlich wurde bei den Experimenten zur Ermittlung geophysikalischer Parameter, daß sich durch eine Versuchsanlage reale Bedingungen nur bedingt simulieren lassen. Die Gefäßwandungen führen zu einer starken Beeinflußung der Stromlinien. Aus diesem Grund wurde bei allen Experimenten dieser Fehler in Kauf genommen, um sich auf die Bestimmung der relativen Änderungen konzentrieren zu können. Dieser Umstand erklärt auch, daß sich die theoretischen Verläufe von spezifischem Widerstand und Potential von den experimentell ermittelten stark unterscheiden.

Die Untersuchungen wurden bevorzugt an Metallelektroden durchgeführt, da diese mit einem minimalen Aufwand in den Boden eingebracht werden können. Deutlich wurde, daß die verschiedenen Elektrodenmaterialien zu unterschiedlichen Potentialverteilungen im Boden führen. Dabei bewirkt eine vergrößerte Elektrodenoberfläche nur eine sehr mäßige Effizienzsteigerung hinsichtlich des Stromflusses.

Bestätigt wurde die Annahme, daß eine ausreichende Feuchte des Bodens für die Ausbildung eines elektrischen Feldes unerläßlich ist, da ansonsten kein Medium vorhanden ist, in dem die

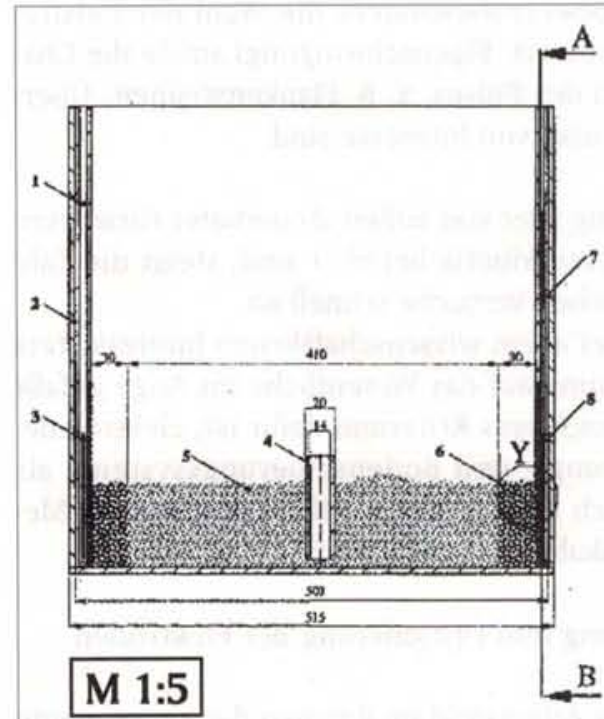

A - B

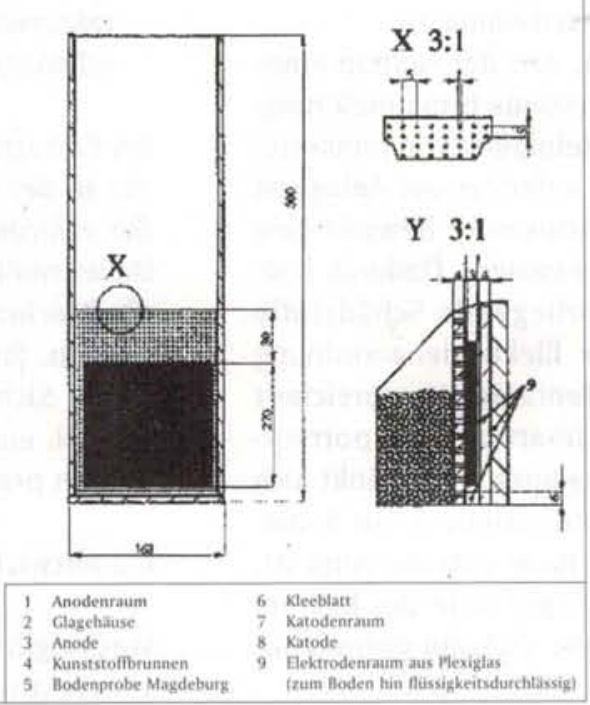
Ladungsträger wandern können. Eine erfolgversprechende Sanierung kann also nur bei einer Abstimmung der Parameter der Elektrosanierung auf die Beschaffenheit des Bodens erfolgen. Bedingt durch den sehr kurzen Projektzeitraum, konnte die Problematik der elektrokinetischen Bodensanierung nur angerissen werden. Um die geophysikalisch meßbaren Parameter mit den tatsächlichen Dekontaminationsvorgängen in Einklang $\mathrm{zu}$ bringen, sind noch eine Reihe von Experimenten notwendig. 
Ergebnisse der Dekontaminationsversuche

Durch die Dekontaminationsversuche konnte bestätigt werden, daß sich bei Einstellung geeigneter Parameter eine Drift der As-Ionen in Richtung Katode einstellt. Dies führt zu einer Anreicherung derselben im Katodenraum.

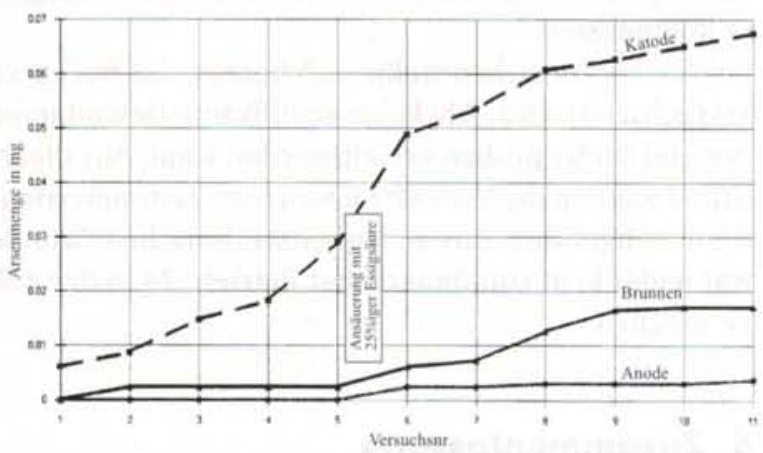

Abb. 5.4.4: Arsenaustrag an Katode, Anode und Brunnen im Laufe der Versuche

Eine pH-Wert Erniedrigung (durch Zugabe von Essigsäure) führt dazu, daß an die Kornmatrix gebundenes As verstärkt in Lösung geht. Die Ansäuerung hat aber auch Einfluß auf die Transportvorgänge im Boden. Es kann dadurch zu einer Einstellung bzw. Umkehrung der Elektroosmose, die neben Elektrolyse und Elektrophorese für den Schadstofftransport verantwortlich ist, kommen.

\section{Gegenwärtiger Stand}

Bei dem durchgeführten Projekt sollte die Entwicklungsproblematik eines elektrokinetischen Sicherungssystems angearbeitet werden.

Entsprechend dem Ziel des beantragten Projekts wurde eine geeignete Versuchsanlage aufgebaut, an der Versuche zur elektrokinetischen Bodensanierung durchgeführt werden können. Neben Versuchen zur Erfassung geophysikalischer Parameter wurden Dekontaminationsversuche an der Anlage durchgeführt. Angestrebt wird die Ermittlung des Zusammenhangs zwischen geophysikalischen Parametern und tatsächlich im Boden stattfindenden chemischen Vorgängen, die zu einem Schadstoffaustrag führen. D. h. es soll eine Verbindung der physikalischen mit der chemischen Seite dieses Verfahrens erarbeitet werden.

Die Interpretation der Ergebnisse der durchgefuihrten Experimente lassen folgende Feststellung zu:

Die Experimente verdeutlichen, daß der Aufbau eines elektrokinetischen Sicherungssystems prinzipiell möglich ist. Eine elektrokinetische Reinigung von feinkörnigen Böden bei entsprechenden Laufzeiten der Anlage ist ebenfalls vorstellbar. Die Elektroosmose bewirkt eine gerichtete Bewegung des Bodenwassers. Dadurch können im Bodenwasser mobil vorliegende Schadstoffe durch eine geeignete Feld- bzw. Elektrodenanordnung in den entsprechenden Elektrodenräumen angereichert werden. Deutlich wurde, daß derartige Transportvorgänge sehr zeitintensiv sind. Dadurch beschränkt sich das Anwendungsfeld der Elektrosanierung auf Sanierungsfälle, bei denen die Dauer nicht entscheidend ist. Da die Sanierungsdauer in der Regel nicht der bestimmende Faktor ist, existieren eine Vielzahl potenieller sanierungsbedürftiger Flächen.

Trotz der langfristigen Sanierungsdauer ist die elektro- kinetische Bodensanierung in einem wirtschaftlichen Rahmen denkbar, da nachgewiesen werden konnte, daß der Aufwand an elektrischer Energie gering ist.

Zwischen den einzelnen Dekontaminationsversuchen kommt es zu einer Diffusion der Schwermetalle zurück in die Bodenmatrix. Aus diesem Grund muß eine kontinuierlich arbeitende Anlage mit einer Elektrodenraumspülung und gekoppelter Spülflüssigkeitsaufbereitung konzipiert werden.

Insbesondere der chemischen Analyse sollte bei folgenden Untersuchungen verstärkte Beachtung geschenkt werden. Bei der photometrischen Bestimmung führt die Wechselwirkung der in der Probe enthaltenen Schwermetalle zu einer Verfälschung der Meßergebnisse. Der As-Schnelltest kann ohnehin nur als abschätzende Methode zur Studie von Effekten herangezogen werden. Aus diesem Grund sind Polarimeter bzw. AAS den bisher verwandten Methoden vorzuziehen.

\section{Ausblick}

\subsection{Fortsetzung der Vorversuche}

Da die Ergebnisse der Experimente nicht in jeder Hinsicht als allgemeingültig anzusehen sind, sondern individuell von der untersuchten Bodenprobe abhängen, sollen die bisherigen Erkenntnisse, soweit möglich, auf weiterfuihrende Versuche mit Bodenproben vom Gelände der TFH Wildau angewendet werden.

Zur Erlangung fundierter, quantitativer Ergebnisse ist die Fortsetzung der Vorversuche dringend erforderlich. Jeweils mit einer Serie gleichartiger Versuche zur systematischen quantitativen Analyse müssen z. B. untersucht werden:

- der Einfluß der Feuchte mit stetig gesteigertem Wasseranteil,

- die Konditionierung des Bodens mit verschiedenen Säuren unterschiedlicher Konzentration,

- der Einfluß von Frequenzen auf die Lösungsbereitschaft bzw. die Mobilisierung der Schwermetallionen. Geplant ist die Verwendung eines gepulsten Gleichstroms, wobei insbesondere die Wahl der Pulsfrequenz (Stichwort: Eigenschwingung) sowie die Charakteristika des Pulses, z. B. Flankensteilheit, Überschwinger usw. von Interesse sind.

Bei Betrachtung aller von außen steuerbarer Parameter, die an der Elektrokinetik beteiligt sind, steigt die Zahl der erforderlichen Versuche schnell an.

Daher muß bei allem wissenschaftlichen Interess stets die Beschränkung auf das Wesentliche ins Auge gefaßt werden. Ein wichtiges Kriterium dafür ist, elektrokinetische Sicherungs- und Bodensanierungssysteme als schnell, einfach und kostengünstig druchführbare Methoden praktikabel zu entwickeln.

\subsection{Entwicklung und Optimierung der Elektroden}

Vordringliches Arbeitsfeld im Rahmen der Experimente zur elektrokinetischen Bodensanierung ist die Entwicklung und Optimierung der Elektroden. Es geht dabei um 
die Erforschung und Effektivierung der vielen Möglichkeiten zur Kombination der Parameter: Elektroden-Material, -Form und -Geometrie, -Anordnung sowie die Konstruktion und Steuerung eines Flüssigkeitskreislaufsystems zum geregelten bzw. regelbaren Austrag der Schadstoffe und Schwermetalle.

Während des Vorgangs der elektrokinetischen Sicherung bzw. Sanierung kann es im Falle zu hoher Spannungen zur teilweisen Auflösung der Anode kommen. Daher sollte und muß das Elektrodenmaterial auch unter ökologischen Aspekten gewählt werden. Mit anderen Worten: bestünde die Anode aus Blei, so würde das Austragen von z. B. Cadmium mit dem Eintrag von Blei in den Boden „bezahlt" werden. Das gäbe keinen Sinn. Unter diesem Blickwinkel ergibt sich eine klare Eingrenzung der als Elektrodenmaterial in Frage kommenden Metalle. Es sollen Stoffe sein, die sowieso in größeren Mengen in natürlichen Böden vorhanden sind. Daher werden die Experimente vornehmlich mit Eisen, Magnesium, Aluminium durchgeführt.

Die Wahl des Elektrodenmaterials muß nicht zuletzt auch getroffen werden unter Berüicksichtigung der Frequenzen und der Form des elektrischen Pulses, sollte sich der Einsatz eines Wechselstroms als günstig erweisen.

\section{Anlagetechnik}

Die erforderliche Technik rund um elektrokinetische Sicherungs- bzw. Sanierungssysteme besteht aus mehreren Komponenten:

- Energiequelle: zur Realisierung mobiler Einheiten ist zunächst ein Generator vorgesehen. Die erforderliche Mindestleistung bzw. seine Dimensionierung hängt von Fall zu Fall ab u. a. von der elektrischen Leitfähigkeit des Untergrundes, von Übergangswiderstand, Material, Bauart und Geometrie der Eletkroden sowie von der Fläche und Tiefe (Volumen) des zu sichernden/ sanierenden Bereichs. In diesem Zusammenhang kann Flurabstand, Mächtigkeit und $\mathrm{K}_{\mathrm{f}}$-Wert des Grundwasserleiters eine entscheidende Rolle spielen.

Später ist die Kopplung mit einer Quelle der sog. alternativen Energien, d. h. regenerativer Energiequellen geplant. Dazu mehr im Abschnitt Vorstellungen über zukünftige Entwicklungen.

- Elektroden: wie oben bereits erwähnt, müssen die günstigsten Eigenschaften für geeignete Elektroden noch durch Testreihen ermittelt werden; m. a. W.: die Elektroden sollen optimiert werden hinsichtlich Form und Material, Oberfläche, Anordnung usw. So sind auch die Konstruktion und der Bau verschiedener Elektroden mit flüssigen Elektrolytbrücken vorgesehen. In Verbindung damit sind Flüssigkeitskreisläufe zu installieren und zu regeln, damit ein kontrollierter Austrag sowie eine kontrollierte Deposition der Schadstoffe gewährleistet ist.

So gesehen ist der technische Aufwand, der unmittelbar mit den Elektroden zusammenhängt, recht groß. Das Ziel ist, eine computergesteuerte Sensorik und Steuerungs- bzw. Regelungstechnik zu entwickeln für die Kontrolle des Zu- und Abstroms von Flüssigkeiten an optimierten Elektroden.
- Kontrollraum: die längerfristige Planung sieht vor, daß die Überwachung des Sicherungs- bzw. Sanierungsablaufs in einem verschließbaren (mobilen) Container stattfinden wird. Darin installiert ist das gesamte technische System zur Sensorik, Regelung und Auswertung.

\section{Anlagenplanung}

Geplant ist die Entwicklung, Installation und Austestung/ Optimierung eines elektrokinetischen Sanierungssystems. Dabei handelt es sich um eine Anlage mit einem überwachten und regelbaren Flüssigkeitskreislauf zur Mehrfachspïlung der zu sanierenden Bodenmassen. Der Schwerpunkt bei der Entwicklung des Sanierungssystems liegt weniger auf dem bereits mehrfach erbrachten Nachweis der Effizienz der Elektrokinetik als auf der Verfahrenstechnik und dem Bau einer optimierten Anlage mit Monitoring-System.

Der Boden befindet sich unter Ausnutzung der Gravitation in einem stufenlos regelbaren vertikal gerichteten elektrischen (Gleichstrom-)Feld. Die Gitter-Katode befindet sich im unteren Teil der Anlage und saugt quasi die mit dem Flïssigkeitsstrom transportierten Schwermetallionen an bzw. entkoppelt sie aus der Bodenmatrix. Einhergehend mit der Entwicklung einer flexibel einsetzbaren mobilen Anlage, die sowohl on-site als auch offsite betrieben werden kann, werden die Fragen zum Flüssigkeitskreislauf sowie zur Entsorgung der Schadstoffe mitbearbeitet. Zur Steigerung der Flexibilität der Anlage ist neben der Regeltechnik hinsichtlich der Durchlaufgeschwindigkeit auch die Umsetzung einer variablen Dimensionierung des Behälters geplant.

\subsection{Anwendungsbeispiel}

Durch den Betrieb solcher Anlagen, z. B. auf dem Standort Wildau, kann der Schadstofftransport in Richtung Wohnansiedlung unmittelbar gestoppt werden. Der Boden wird kontinuierlich fortschreitend elektrokinetisch bearbeitet. Nach Unterschreiten der vorgegebenen Grenzwerte (Brandenburger Liste) ist der Boden on-site saniert; ein Abtransport und eine kostenintensive Endlagerung auf einer Sondermuilldeponie ist nicht erforderlich. Der Forschungs- und Lehrbetrieb auf dem kontaminierten Gelände der TFH Wildau kann quasi ungestört fortgefuihrt werden; erforderliche Bodenaushubarbeiten finden nur lokal statt.

\subsection{Wirtschaftliche Zielstellung und Einsetzbarkeit elektrokinetischer Sicherungs- und Sanierungs- systeme}

In der Einleitung wurden bereits einige Anwendungsgebiete der Elektrokinetik ohne Anspruch auf Vollständigkeit plakativ aufgelistet. An dieser Stelle soll noch einmal betont sein, daß die Entwicklung einer mobilen Sanierungsanlage mit großen Vorteilen verbunden ist. Sie kann in kürzester Zeit an jedem Ort auch zur Vorfuihrung und Überzeugung potentieller Auftraggeber in Betrieb gesetzt werden. 
Langwierige Genehmigungsverfahren, wie sie für den Betrieb größerer stationärer Anlagen zu erwarten sind, können unter dem Aspekt der kleineren Dimensionierung und des zeitlich begrenzten Einsatzes der mobilen Anlage erheblich gestrafft werden.

Ein ganz wesentlicher Vorteil ist der netzunabhängige Betrieb der Anlage. Die Energie-Erzeugung erfolgt vor Ort autark z. B. per Generator. Für die zukünftige Weiterentwicklung des Projekts ist die Energieversorgung mittels einer ebenfalls mobilen Holzvergasungsanlage vorgesehen.

Die TFH Wildau steht in engem Kontakt zu gewerblichen Partnern, die sich seit Jahren intensiv mit der biologischen Reinigung/Sanierung von Böden auseinandersetzen. Gespräche über die Möglichkeiten der Kopplung von elektrokinetischer und biologischer Bodensanierung fanden bereits statt. Ein Aspekt hier ist z. B., daß die moderate Aufheizung des Bodens mit einer positiven Wirkung für die Vermehrung der Bakterien verbunden ist. Das elektrische Feld wird von den Einzellern aufgrund ihrer Dimension nicht wahrgenommen.

Größere stationäre elektrokinetische Sanierungsanlagen können/sollten zur Vorbehandlung bei der Verbrennung von Klärschlamm und/oder im Vorfeld ihrer Deponierung eingesetzt werden.

\subsection{Vorstellungen über die zukünftige Entwicklung}

Die Grundgesetze der Physik und Chemie, die Literatur, aber auch die ersten eigenen Erfahrungen weisen darauf hin, daß auch die Abläufe in der Elektro-Chemie durch Druck- und Temperaturerhöhung gesteuert und beschleunigt bzw. effektiviert werden können. Folgerichtig ergibt sich daraus für die Zukunft die Idee, das elektrokinetische Sicherungs- bzw. Sanierungssystem zu koppeln mit flankierenden thermischen und hydraulischen Maßnahmen.

Es soll weiterführend eine interdisziplinäre elektrokinetische Sanierungs- und Sicherungstechnik entwikkelt werden. Dabei wird an eine gesteuerte Durchflutung des Untergrundes mit einer heißen, umweltverträglichen, verdünnten Säure gedacht. Für die Durchfuihrung derartiger Maßnahmen ist die Einbeziehung und Berücksichtigung von Erfahrungen, die mit hydraulischen Bodenwaschanlagen gemacht worden sind, unbedingt zu empfehlen. Die wichtigsten beteiligten Fachgebiete werden sein:

Physik, Elektronik, Informatik, Physikalische Chemie, Technische Chemie, Geophysik, Geologie, Hydrologie, Umwelttechnik.

Die zu bearbeitenden Problemfelder werden sein:

- Energieversorgung: Strom und Wärme

- Elektroden: Form, Material, Oberfläche, Bauart, Anordnung

- Wasseraufbereitung: Härtegrad, Erwärmung, Zusätze, Durchmischung

- Hydraulik/Druck: Einpressen und Entnahme der Flïssigkeiten

- Entgasungstechnik: Auffangen, Analysieren, Binden aufsteigender Gase
- Entsorgung: Filtern und Reinigen von Flüssigkeiten, Trennung, Sammlung \& Recycling von Abscheidungen, Reduktion, Entlagerung

- Sicherheitstechnik: Abschirmung giftiger Substanzen (Gase), Schutzkleidung

\section{Verfasser}

Prof. Dr.-Ing. Udo Hellwig

Technische Fachhochschule Wildau

Fachbereich Verfahrenstechnik

Tel. (0 33 75) 508-393/-392 